\title{
A REMARK ON THE BRYLINSKI CONJECTURE FOR ORBIFOLDS
}

\author{
L. BAK and A. CZARNECKI ${ }^{凶}$ \\ (Received 21 February 2010; accepted 11 August 2011) \\ Communicated by C. D. Hodgson
}

\begin{abstract}
The paper presents a proof of the Brylinski conjecture for compact Kähler orbifolds. The result is a corollary of the foliated version of the Mathieu theorem on symplectic harmonic representations of de Rham cohomology classes. The proofs are based on the idea of representing an orbifold as the leaf space of a Riemannian foliation and on the correspondence between foliated and holonomy invariant objects for foliated manifolds.
\end{abstract}

2010 Mathematics subject classification: primary 53D05; secondary 53C12, 57R18.

Keywords and phrases: transversally symplectic foliations, symplectic orbifolds, Brylinski conjecture, Mathieu theorem.

\section{Introduction}

The goal of this paper is to prove an orbifold version of the Brylinski conjecture [2], which aimed to establish a symplectic counterpart of the classical Hodge theory and was concerned with the question whether every cohomology class on compact symplectic manifolds admits a symplectically harmonic representative. Brylinski proved that this is the case for some interesting classes of manifolds, most notably for compact Kähler manifolds, but in general the conjecture does not hold. The first to present counterexamples were Mathieu [12] and, independently, Fernández et al. [6]. Moreover, Mathieu gave an equivalent condition, in terms of the cohomological properties of the symplectic form, for a symplectic manifold (not necessarily compact) to satisfy the conjecture. The same question about the existence of symplectically harmonic representatives can be stated for symplectic orbifolds and, in particular, for compact Kähler orbifolds. The main result of this paper is the following theorem.

TheOReM 1. The Brylinski conjecture holds for compact Kähler orbifolds.

The study of the homological properties of singular spaces tends to be more complicated than that for manifolds. Many properties that prove very useful in the

(C) 2011 Australian Mathematical Publishing Association Inc. 1446-7887/2011 \$16.00 
smooth setting such as Poincaré duality and the finite dimension of homology groups do not hold in general. These problems have been addressed in different ways. One approach is to refine the homology theory in question to take singularities into account. This approach led to the definition of new homology theories, such as Goresky and MacPherson's intersection homology and cohomology [8]. For orbifolds, which are of interest to us in this paper, another method is applicable.

It is well known (see [14]) that the space of leaves of a Riemannian foliation of a compact manifold with compact leaves is an orbifold. In [7] the converse was proved.

THEOREM 2. Every orbifold can be realized as the space of leaves of a Riemannian foliation.

Their proof is written for complex orbifolds and transversally holomorphic foliations but it can be adapted to the real case. This construction, despite its usefulness for research into the properties of orbifolds, has not been fully exploited. The construction was recently used by Wang and Zaffran [21] to give a simple proof of the hard Lefschetz theorem for Kähler orbifolds, a fact that will be useful to us.

Prior to [21], only a few other authors took up the similar idea of applying foliated Riemannian manifolds and their foliated objects to the study of the geometry of the leaf (closure) space (see [1, 10, 16]). The realization of an orbifold as the space of leaves of a Riemannian foliation allows us to consider 'transverse' objects on a foliated manifold rather than the corresponding objects on an orbifold. Our next step is to reformulate the problem again, this time using the well-known correspondence between 'transverse' objects on foliated manifolds and 'holonomy invariant' objects on the corresponding transverse manifolds. We will only sketch this correspondence in the scope needed for this paper. A more general and exhaustive approach can be found, for example, in [22]. Eventually we shall see that Theorem 1 is a corollary of the following theorem.

THEOREM 3. Let $M$ be a manifold and let $\Gamma$ be a pseudogroup of local diffeomorphisms of $M$. Let $\omega$ be a $\Gamma$-invariant symplectic form on $M$. Then the following conditions are equivalent.

(1) Every $\Gamma$-invariant cohomology class has a harmonic representative.

(2) For each $k \in\{0,1, \ldots, n\}$, the mapping $L^{k}: H_{\Gamma}^{n-k}(M) \rightarrow H_{\Gamma}^{n+k}(M)$, where $L[\xi]=$ $[\omega \wedge \xi]$, is surjective.

In Section 2 we recall some basic constructions concerning the complex of invariant differential forms and prove Theorem 3. In Section 3 we deduce a foliated version of Mathieu's result from Theorem 3. In Section 4 we consider a special structure that combines a transversally symplectic form and leafwise metric and generalize Pak's result [15] about transversally harmonic forms on manifolds with a transversally symplectic flow. Finally in Section 5 we prove Theorem 1.

\section{Invariant forms}

In this section we shall consider a smooth manifold $M$ of dimension $m$ together with a pseudogroup of local diffeomorphisms $\Gamma$. From the complex of differential 
forms $\Omega^{*}(M)$ we single out the $\Gamma$-invariant forms, that is, those $\xi \in \Omega^{*}(M)$ satisfying the condition

$$
\left.\xi\right|_{U}=\gamma^{*}\left(\left.\xi\right|_{\gamma(U)}\right) \quad \forall \gamma \in \Gamma
$$

where $U$ is the domain of $\gamma$. These differential forms form a subcomplex (which we shall denote by $\Omega_{\Gamma}^{*}(M)$ ) with differential $d=\left.d\right|_{\Omega_{\Gamma}^{*}(M)}$. The homology of this complex is called $\Gamma$-invariant cohomology and is denoted by $H_{\Gamma}^{*}(M)$. In a similar manner we can define the $\Gamma$-invariant multivector fields to be those $X \in \mathfrak{X}^{*}(M)$ satisfying the condition

$$
\left.X\right|_{\gamma(U)}=\gamma_{*}\left(\left.X\right|_{U}\right) \quad \forall \gamma \in \Gamma .
$$

We shall use the notation $\mathfrak{X}_{\Gamma}^{*}(M)$ to denote the space of $\gamma$-invariant multivector fields.

For $k<l$ we have a natural nondegenerate pairing

$$
\mathfrak{X}^{k}(M) \times \Omega^{l}(M) \ni(X, \xi) \mapsto \iota_{X} \xi \in \Omega^{l-k}(M) .
$$

Direct computations show that this pairing restricts to a pairing

$$
\mathfrak{x}_{\Gamma}^{k}(M) \times \Omega_{\Gamma}^{l}(M) \rightarrow \Omega_{\Gamma}^{l-k}(M) .
$$

We may use a $\Gamma$-invariant volume form to obtain an isomorphism

$$
\mathfrak{X}_{\Gamma}^{k}(M) \cong \Omega_{\Gamma}^{m-k}(M)
$$

as follows. From now on we shall assume that $M$ is of even dimension $m=2 n$. Note that a $\Gamma$-invariant symplectic form is just a closed, nondegenerate element $\omega \in \Omega_{\Gamma}^{2}(M)$. From this we may deduce that the form $\omega^{n}$ is a $\Gamma$-invariant volume form and the isomorphism (1) follows. On the other hand, we have an isomorphism

$$
\mathfrak{X}_{\Gamma}(M) \ni X \mapsto \iota_{X} \omega \in \Omega_{\Gamma}^{1}(M)
$$

which extends to an algebra isomorphism

$$
\mathfrak{X}_{\Gamma}^{*}(M) \cong \Omega_{\Gamma}^{*}(M) .
$$

Combining these two isomorphisms we obtain an isomorphism

$$
\star: \Omega_{\Gamma}^{*}(M) \cong \mathfrak{X}_{\Gamma}^{*}(M) \cong \Omega_{\Gamma}^{2 n-*}(M)
$$

associated with the symplectic structure $\omega$.

REMARK 4. The isomorphism $\star$ satisfies the condition $\star^{2}=\operatorname{id}_{\Omega_{\Gamma}^{*}(M)}$.

We now consider the codifferential $\delta$ given, for any $\xi \in \Omega_{\Gamma}^{k}(M)$, by

$$
\delta \xi=(-1)^{k} \star d \star \xi .
$$

It follows easily from the above properties that $\delta^{2}=0$. The central idea in our further studies will be the notion of a harmonic $\Gamma$-invariant form $\xi \in \Omega_{\Gamma}^{*}(M)$ such that $d \xi=0$ and $\delta \xi=0$. Forms satisfying these conditions constitute a subcomplex of $\Omega_{\Gamma}^{*}(M)$, which we shall denote by $\Omega_{\Gamma, h}^{*}(M)$. 
The proof of Theorem 3 will closely follow Yan's proof [23] of Mathieu's result. We now recall Yan's method. We omit some calculations that are identical to those given in [23].

We construct a Lie algebra $\mathfrak{s l}(2)$-representation on $\Omega_{\Gamma}^{*}(M)$. We represent the usual basis of $\mathfrak{s l}(2)$ given by

$$
X=\left[\begin{array}{ll}
0 & 1 \\
0 & 0
\end{array}\right], \quad Y=\left[\begin{array}{ll}
0 & 0 \\
1 & 0
\end{array}\right], \quad H=\left[\begin{array}{cc}
1 & 0 \\
0 & -1
\end{array}\right]
$$

as operators on $\Omega_{\Gamma}^{*}(M)$ (denoted by the same letters) so that the relations $[X, Y]=H$, $[H, X]=2 X$ and $[H, Y]=-2 Y$ hold. We set

$$
\begin{aligned}
& Y \leadsto \Omega_{\Gamma}^{k}(M) \ni \xi \mapsto \omega \wedge \xi \in \Omega_{\Gamma}^{k+2}(M), \\
& X \leadsto \Omega_{\Gamma}^{k}(M) \ni \xi \mapsto \star Y \star \xi \in \Omega_{\Gamma}^{k-2}(M), \\
& H \leadsto \Omega_{\Gamma}^{k}(M) \ni \xi \mapsto(n-k) \xi \in \Omega_{\Gamma}^{k}(M) .
\end{aligned}
$$

Observe that the operator $L$ from the statement of Theorem 3 is induced by $Y$. Eigenvectors of $H$ from the kernel of $X$ are called primitive. We say that an sl(2)representation on some vector space $V$ is of finite $H$-spectrum if and only if this space splits into a finite sum of the eigenspaces of the operator $H$. This is obviously the case for our representation. The following properties hold for $\mathfrak{s l}(2)$-representations of finite $H$-spectrum (see $[9,23]$ ).

Proposition 5. Let $V$ be a vector space with an sl(2)-representation of finite $H$ spectrum. Let $V_{\lambda}$ be the $\lambda$-eigenspace of $H$ and let $P_{\lambda}$ stand for the set of primitive elements of $V_{\lambda}$. Then:

(1) all eigenvalues of $H$ are integers;

(2) $\left.X\right|_{V_{k}}: V_{k} \rightarrow V_{k+2},\left.Y\right|_{V_{k}}: V_{k} \rightarrow V_{k-2}$ for each $k \in \mathbb{Z}$;

(3) $\quad X^{k} \mid V_{-k}: V_{-k} \rightarrow V_{k}$ and $Y^{k} \mid V_{k}: V_{k} \rightarrow V_{-k}$ are isomorphisms for each $k \in \mathbb{N}$;

(4) $P_{k}=\left\{v \in V_{k} \mid Y^{k+1} v=0\right\}$;

(5) every $V_{k}$ admits a direct sum decomposition of the form $V_{k}=\left.P_{k} \oplus \operatorname{Im} Y\right|_{V_{k+2}}$.

We apply this proposition in our case to obtain the following corollary.

Corollary 6. The operator

$$
Y^{k}: \Omega_{\Gamma}^{n-k}(M) \rightarrow \Omega_{\Gamma}^{n+k}(M)
$$

is an isomorphism.

The next step is to prove that this representation on $\Omega_{\Gamma}^{*}(M)$ induces a representation on the subspace of $\Gamma$-invariant harmonic forms $\Omega_{\Gamma, h}^{*}(M)$ or, equivalently, that $X, Y$ and $H$ preserve the harmonic forms. This follows from the identities

$$
[Y, d]=[X, \delta]=0, \quad[X, d]=-\delta, \quad[Y, \delta]=-d, \quad[H, d]=[H, \delta]=0 .
$$


Corollary 7. The operator

$$
Y^{k}: \Omega_{\Gamma, h}^{n-k}(M) \rightarrow \Omega_{\Gamma, h}^{n+k}(M)
$$

is an isomorphism.

Proof of Theorem 3. We are now ready to prove the implication from (1) to (2) of Theorem 3 . The following diagram is clearly commutative

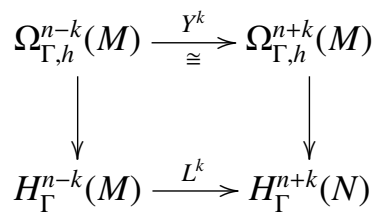

with natural vertical arrows. If we assume the surjectivity of the vertical arrows, then we obtain the surjectivity of the lower horizontal arrow, as required. We will use the following lemma, which is a simple corollary of the relation $\delta=-[X, d]$ in the proof of the converse.

Lemma 8. A form $\xi \in \Omega_{\Gamma}^{*}(M)$ that is closed and primitive must be harmonic.

Now we assume condition (2) of Theorem 3 and proceed with the proof of condition (1) of Theorem 3 by induction. Each closed 0-form is clearly harmonic. It follows by Lemma 8 that every closed 1 -form is harmonic.

Let $k$ be such that $n-k \geq 2$ and Theorem 3(1) holds for every cohomology class of degree $r<n-k$. Let $\xi \in \Omega_{\Gamma}^{n-k}(M)$ be a closed form. It suffices to prove that $\xi$ is cohomologous to some harmonic form. By Theorem 3(2) there exist a closed form $\eta \in \Omega_{\Gamma}^{n-k-2}(M)$ and a form $\theta \in \Omega_{\Gamma}^{n+k+1}(M)$ such that

$$
\omega^{k+2} \wedge \eta+d \theta=\omega^{k+1} \wedge \xi
$$

Now $\eta$ is cohomologous to some harmonic form $\bar{\eta} \in \Omega_{\Gamma, h}^{n-k-2}(M)$ and so $\eta=\bar{\eta}+d \lambda$ for some $\lambda \in \Omega_{\Gamma}^{n-k-3}(M)$. We know that the map

$$
Y^{k+1}: \Omega_{\Gamma}^{n-k-1}(M) \rightarrow \Omega_{\Gamma}^{n+k+1}(M)
$$

is surjective and so we can choose $\zeta \in \Omega_{\Gamma}^{n-k-1}(M)$ satisfying $\omega^{k+1} \wedge \zeta=\theta$. Finally we may deduce that

$$
\omega^{k+1} \wedge[\xi-d(\zeta+\omega \wedge \lambda)-\omega \wedge \bar{\eta}]=0 .
$$

The form $\bar{\xi}=\xi-d(\zeta+\omega \wedge \lambda)$ is cohomologous to $\xi$. By part (4) of Proposition 5, $\bar{\xi}-\omega \wedge \bar{\eta}$ is primitive. It follows by Lemma 8 that $\bar{\xi}-\omega \wedge \bar{\eta}$ is harmonic. However, $\omega \wedge \bar{\eta}$ is harmonic. The harmonicity of $\bar{\xi}$ follows. 


\section{Foliations}

Let $M$ be a manifold with a regular foliation $\mathcal{F}$ of dimension $p$ and even codimension $2 n$. If $U \subset M$ is a sufficiently small open set, then there is a submersion $p: U \rightarrow \mathbb{R}^{2 n}$ such that the foliation $\mathcal{F}$ restricted to $U$ is given by the fibers of $p$. Let $\left\{U_{i}\right\}$ be an atlas on $M$ consisting of open sets admitting submersions $p_{i}: U_{i} \rightarrow \mathbb{R}^{2 n}$ as above. Using this atlas we can associate the transverse manifold $N=\amalg p_{i}\left(U_{i}\right)$ with the holonomy pseudogroup $\Gamma$ generated by the mappings from the associated Haefliger cocycle (see [13]).

Consider the basic complex $\Omega_{B}^{*}(M, \mathcal{F})$ consisting of the basic forms $\xi$, which satisfy the condition $\iota_{T} \xi=\mathcal{L}_{T} \xi=0$ for each vector field $T$ tangent to the foliation. The cohomology of this complex is also called basic and is denoted by $H_{B}^{*}(M, \mathcal{F})$. Intuitively, basic forms are those that are locally pullbacks of forms from the transverse manifold. This intuitive description is justified by the following observation.

Proposition 9. There is a chain isomorphism $\Xi: \Omega_{B}^{*}(M, \mathcal{F}) \stackrel{\cong}{\longrightarrow} \Omega_{\Gamma}^{*}(N)$ satisfying

$$
\left.\xi\right|_{U_{i}}=p_{i}^{*}\left(\left.\Xi(\xi)\right|_{p_{i}\left(U_{i}\right)}\right)
$$

for every $\xi \in \Omega_{B}^{*}(M, \mathcal{F})$. Moreover $\Xi$ is an algebra homomorphism.

Fix a transversally symplectic structure on $M$, that is, a basic, closed, nondegenerate 2 -form $\omega$. By Proposition 9, $\omega$ induces a $\Gamma$-invariant symplectic structure on $N$, namely $\Xi(\omega)$.

Consider the mapping

$$
\mathfrak{X}(M) \ni X \mapsto \iota_{X} \omega \in \Omega^{1}(M) .
$$

To make this mapping an isomorphism we shall restrict it to the basic vector fields, which are defined as follows. Let $\mathcal{D}$ be an arbitrary distribution in $T M$ complementary to $T \mathcal{F}$. The space of basic vector fields $\mathfrak{X}_{B}(M, \mathcal{F})$ is given by

$$
\mathfrak{X}_{B}(M, \mathcal{F})=\{X \in \mathfrak{X}(\mathcal{D}) \mid[X, T] \in \mathfrak{X}(\mathcal{F}) \forall T \in \mathfrak{X}(\mathcal{F})\}
$$

where $\mathfrak{X}(\mathcal{F})$ denotes those vector fields tangent to the foliation and $\mathfrak{X}(\mathcal{D})$ denotes those with values in $\mathcal{D}$. It is a simple calculation to check that

$$
\mathfrak{X}_{B}(M, \mathcal{F}) \ni X \mapsto \iota_{X} \omega \in \Omega_{B}^{1}(M, \mathcal{F})
$$

is a well-defined isomorphism. If we take the subalgebra generated by basic vector fields in $\mathfrak{X}^{*}(M)$ and denote it by $\mathfrak{X}_{B}^{*}(M, \mathcal{F})$, then this isomorphism extends to an isomorphism $\mathfrak{X}_{B}^{*}(M, \mathcal{F}) \cong \Omega_{B}^{*}(M, \mathcal{F})$.

On the other hand we can show that

$$
\mathfrak{X}_{B}^{*}(M, \mathcal{F}) \ni X \mapsto \iota_{X} \omega^{n} \in \Omega_{B}^{2 n-*}(M, \mathcal{F})
$$

is, again by simple calculations, an isomorphism, and we obtain the transversally symplectic star operator

$$
\star_{t}: \Omega_{B}^{*}(M, \mathcal{F}) \cong \mathfrak{X}_{B}^{*}(M, \mathcal{F}) \cong \Omega_{B}^{2 n-*}(M, \mathcal{F}) .
$$


Observe that while both of the isomorphisms that we have defined depend on the choice of a distribution $\mathcal{D}$, their composition does not. Having a star-operator at our disposal, we proceed in the usual manner. We define a codifferential $\delta_{t} \xi=(-1)^{k} \star_{t} d \star_{t}$ for $\xi \in \Omega_{B}^{k}(M, \mathcal{F})$ and consider the forms $\xi$ for which $d \xi=\delta_{t} \xi=0$, namely the harmonic forms. The reader should bear in mind that, despite the similar language, the symplectic setting presented here differs greatly from the foliated Hodge theory introduced in [5].

The following lemma and the chain isomorphism $\Xi$ permit us to apply Theorem 3 in our investigations of the basic case.

LEMMA 10. The following diagram is commutative.

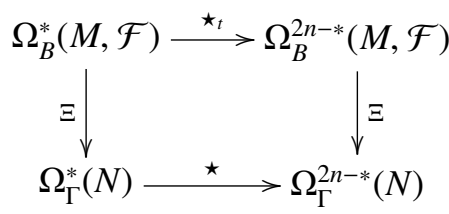

This assures that $\Xi$ induces a correspondence between the basic harmonic forms on $M$ and the $\Gamma$-invariant harmonic forms on $N$. Now we can apply Theorem 3 to obtain the following result.

THEOREM 11. The following conditions are equivalent.

(1) Every basic cohomology class has a harmonic representative.

(2) For each $k \in\{0,1, \ldots, n\}$ the mapping

$$
L^{k}: H_{B}^{n-k}(M, \mathcal{F}) \rightarrow H_{B}^{n+k}(M, \mathcal{F})
$$

is surjective.

\section{Harmonicity with respect to the metric and symplectic structure}

Pak [15] considered the question of the existence of harmonic forms when the operator $\star$ is defined with respect not only to the transversal structure of a dimension one foliation but also to some leafwise structure. To avoid confusion we shall denote this new operator by $\star_{a}$. This approach seems to be proper if we want to define harmonicity on the whole manifold and not only on the transverse structure. We shall now reformulate this problem for foliations of arbitrary dimension.

Recall that $M$ is a manifold with a regular foliation $\mathcal{F}$ of dimension $p$ and even codimension $2 n$ with transversally symplectic structure $\omega \in \Omega_{B}^{2}(M, \mathcal{F})$. Assume that the leaves of this foliation are orientable or, equivalently, that $M$ is orientable. Fix a metric $g$ on $M$. The tangent bundle $T M$ splits into the direct sum $T M=T \mathcal{F} \oplus T \mathcal{F}^{\perp}$. This splitting induces a bigrading of the space of forms

$$
\Omega^{*}(M)=\bigoplus_{k=0}^{p+2 n} \Omega^{k}(M)=\bigoplus_{k=0}^{p+2 n} \bigoplus_{r, s \geq 0, r+s=k} \Omega^{r, s}(M, \mathcal{F}) .
$$


Note that $\xi \in \Omega^{r, s}(M, \mathcal{F})$, that is, $\xi$ is of type $(r, s)$ if and only if

$$
\iota_{X_{1} \wedge \cdots \wedge X_{r+1}} \xi=\iota_{Y_{1} \wedge \cdots \wedge Y_{s+1}} \xi=0
$$

for $X_{1}, \ldots, X_{r+1} \in \mathfrak{X}(\mathcal{F})$ and $Y_{1}, \ldots, Y_{s+1} \in \mathfrak{X}^{\perp}(\mathcal{F})$. Clearly the basic $k$-forms are of type $(0, k)$.

The orientability of leaves allows us to choose a leafwise volume form $\chi$ of type $(p, 0)$ with respect to $g$. The form $\omega^{n} \wedge \chi$ is a volume form on $M$ and we have an isomorphism

$$
\mathfrak{X}^{*}(M) \ni X \mapsto \iota_{X}\left(\omega^{n} \wedge \chi\right) \in \Omega^{p+2 n-*}(M) .
$$

As we have done earlier, we define an isomorphism $\mathfrak{X}(M) \rightarrow \Omega^{1}(M)$, denoted by $b$ and given by

$$
b(X)=\iota_{X^{v}} \omega+g\left(X^{h}, \cdot\right)
$$

where $X=X^{h}+X^{v}$ and $X^{h}, X^{v}$ belong to $\mathfrak{X}(\mathcal{F})$ and $\mathfrak{X}^{\perp}(\mathcal{F})$, respectively. We then extend our isomorphism to

$$
b: \mathfrak{X}^{*}(M) \rightarrow \Omega^{*}(M) .
$$

The star operator obtained as the usual composition

$$
\star_{a}: \Omega^{*}(M) \cong \mathfrak{X}^{*}(M) \cong \Omega^{p+2 n-*}(M)
$$

depends on the choice of the metric $g$. We will be interested in the behaviour of this operator restricted to basic forms.

Consider the bigrading $\mathfrak{X}^{*, *}(M)$ such that $(r+s)$-vector field $X$ is of type $(r, s)$ if and only if $\iota_{X} \xi=0$ for each $(r+s)$-form $\xi$ of type other than $(r, s)$. We can now rewrite (2) as

$$
\star_{a}: \Omega^{*, *}(M, \mathcal{F}) \cong \mathfrak{X}^{*, *}(M) \cong \Omega^{p-*, 2 n-*}(M) .
$$

For a basic $k$-form $\xi$ we calculate that $b^{-1}(\xi)$ is of type $(0, k)$ and therefore

$$
\star_{a} \xi=\iota_{b^{-1}(\xi)}\left(\omega^{n} \wedge \chi\right)=\iota_{b^{-1}(\xi)} \omega^{n} \wedge \chi=\star_{t} \xi \wedge \chi .
$$

If we consider the codifferential defined for $k$-forms $\xi$ by $\delta_{a} \xi=(-1)^{k} \star_{a} d \star_{a} \xi$, then for a basic $k$-form we obtain

$$
\begin{aligned}
\delta_{a} \xi & =(-1)^{k} \star_{a} d \star_{a} \xi \\
& =(-1)^{k} \star_{a} d\left(\star_{t} \xi \wedge \chi\right) \\
& =\star_{a}\left((-1)^{k} d \star_{t} \xi \wedge \chi+\star_{t} \xi \wedge d \chi\right) \\
& =\iota_{(-1)^{k} b^{-1}\left(d \star_{t} \xi\right) \wedge b^{-1}(\chi)}\left(\omega^{n} \wedge \chi\right)+\iota_{b^{-1}\left(\star_{t} \xi\right) \wedge b^{-1}(d \chi)}\left(\omega^{n} \wedge \chi\right) \\
& =\iota_{b^{-1}(\chi)}\left(\delta_{t} \xi \wedge \chi\right)+\iota_{b^{-1}(d \chi)}\left(\star_{t}^{2} \xi \wedge \chi\right) \\
& =(-1)^{p(k-1)} \delta_{t} \xi+\iota_{b^{-1}(d \chi)}(\xi \wedge \chi) .
\end{aligned}
$$

Observe that the differential $d$ splits into three parts

$$
d=d_{1,0}+d_{0,1}+d_{-1,2}
$$


with respect to the bigrading $\Omega^{*, *}(M)$. In particular, $d \chi=d_{0,1} \chi+d_{-1,2} \chi$ since $d_{1,0} \chi$ vanishes. In general the summand $d_{-1,2 \chi}$ does not vanish and thus $\delta_{a} \xi$ is not basic when $\xi$ is a basic form. Therefore we must redefine the codifferential as

$$
\delta_{a} \xi=(-1)^{k} \star_{a} d_{0,1} \star_{a} \xi .
$$

Under this definition the codifferential is the adjoint of $d_{0,1}$ instead of $d$, but on basic forms these two operators coincide.

Now if we recall that $d_{0,1} \chi=\kappa \wedge \chi$ where $\kappa$ is the mean curvature form for $\mathcal{F}$ and write $T=b^{-1}(\kappa)$, then we see that

$$
\delta_{a} \xi=(-1)^{p(k-1)}\left(\delta_{t} \xi+\iota_{T} \xi\right) .
$$

So for basic forms to be preserved by the codifferential we must assume that $\kappa$ is basic. Foliations with this property are called tense.

We recall the operator $Y$ defined in Section 2 as follows:

$$
Y: \Omega_{B}^{*}(M, \mathcal{F}) \ni \xi \mapsto \omega \wedge \xi \in \Omega_{B}^{*}(M, \mathcal{F}) .
$$

By Lemma 10 we may deduce that $\left[Y, \delta_{t}\right]=-d$. We now compute $\left[Y, \delta_{a}\right] \xi$ for a basic $k$-form $\xi$.

$$
\begin{aligned}
{\left[Y, \delta_{a}\right] \xi } & =Y \delta_{a} \xi-\delta_{a} Y \xi=(-1)^{p(k-1)}\left(\left[Y, \delta_{t}\right] \xi+Y \iota_{T} \xi-\iota_{T} Y \xi\right) \\
& =(-1)^{p(k-1)+1}\left(\iota_{T} \omega \wedge \xi+d \xi\right) .
\end{aligned}
$$

This means that in order to obtain the invariance of basic forms under $Y$ we need to assume that $\iota_{T} \omega=0$. However, the nondegeneracy of $\omega$ then implies that the mean curvature vanishes. In this case the basic forms harmonic with respect to $\star_{a}$ and the basic forms harmonic with respect to $\star_{t}$ coincide because $\star_{a}=\star_{t}$. We have now proved the following theorem.

THeorem 12. Let $\mathcal{F}$ be a foliation with vanishing mean curvature form $\mathrm{\kappa}$. Then the following conditions are equivalent.

(1) Every basic cohomology class has a harmonic representative with respect to the operator $\star_{a}$.

(2) For each $k \in\{0,1, \ldots, n\}$ the mapping

$$
L^{k}: H_{B}^{n-k}(M, \mathcal{F}) \rightarrow H_{B}^{n+k}(M, \mathcal{F})
$$

is surjective.

Using a well-known technique, we can deform the metric along the leaves to change the mean curvature form inside its cohomology class. In particular, if we can find a metric on a manifold for which the mean curvature form is exact, then we can find another metric with vanishing mean curvature. In the case of a tense, transversally oriented Riemannian foliation on a closed oriented manifold, the exactness of the mean curvature form is equivalent to the minimalizability of the leaves (see [20]). The work of [4] enables us to omit the tenseness condition. Recall that we consider only foliations with orientable leaves. We can now state the following corollary. 
COROLlary 13. For a transversally symplectic, minimalizable Riemannian foliation on a closed manifold there exists a metric for which conditions (1) and (2) of Theorem 12 are equivalent.

Masa [11] proved that for a transversally orientable Riemannian foliation on a closed, orientable manifold, the minimalizability of leaves is equivalent to the nontriviality of the highest rank basic cohomology. Thus we obtain another result.

Corollary 14. For a transversally symplectic Riemannian foliation of codimension $2 n$ on a closed manifold such that $H_{B}^{2 n}(M, \mathcal{F}) \neq 0$ there exists a Riemannian metric for which conditions (1) and (2) of Theorem 12 are equivalent.

In addition, Rummler [17] proved that a Riemannian foliation with compact leaves is minimalizable and so we may deduce the next corollary.

Corollary 15. For a transversally symplectic Riemannian foliation of codimension $2 n$ with compact leaves on a closed manifold there exists a Riemannian metric for which conditions (1) and (2) of Theorem 12 are equivalent.

\section{Orbifolds}

The notion of an orbifold is a generalization of the notion of a manifold. While a manifold locally looks like a Euclidean space, an orbifold locally looks like a quotient of a Euclidean space under an action of a finite group. The class of orbifolds includes not only manifolds, but also manifolds with boundary and with corners. The first definition of an orbifold was given by Satake [18] and referred to as a $V$-manifold. Numerous other definitions were given later, among them, by Thurston [19] and by Moerdijk and Mrčun [13]. In this note we follow the definitions given by Chen and Ruan [3] using local uniformizing systems.

A section of the bundle of positive, symmetric tensors of type $(0,2)$ on an orbifold $X$ plays the role of a Riemannian metric on $X$ and any orbifold $X$ admits such a metric. If we choose a Riemannian metric on $X$, then we can consider the bundle of orthonormal frames. For any uniformizing system $\left\{\left(V_{i}, \mathcal{G}_{i}, \pi_{i}\right)\right\}$ where $V_{i}$ is a Riemannian manifold the group $\mathcal{G}_{i}$ is a finite group acting effectively by isometries on $V_{i}$ and $\pi_{i}: V_{i} \rightarrow U_{i}$ is a $\mathcal{G}_{i}$-invariant projection onto some open set $U_{i} \subset X$, which induces a homeomorphism between $V_{i} / G$ and $U_{i}$.

The action of $\mathcal{G}_{i}$ lifts in a natural way to a free action on $L V_{i}$, which is the bundle of orthonormal frames over $V_{i}$. The manifolds $L V_{i} / \mathcal{G}_{i}$ can be glued together to form a manifold $L V$ on which $O(n)$ acts, giving it the structure of a foliated manifold. The corresponding space of leaves has a natural orbifold structure, which is isomorphic to $X$. For details of this construction see [13]. The basic forms of this foliation are exactly the forms on the orbifold $X$. In particular, if we fix a symplectic structure on $X$, then we obtain a basic symplectic form on the associated foliated manifold. Other constructions from Section 3 can also be performed on $X$ and we may deduce the following corollary of Theorem 11. 
THeOREM 16. For a symplectic orbifold $X$ of dimension $n$ the following conditions are equivalent.

(1) Every cohomology class has a harmonic representative.

(2) For each $k \in\{0,1, \ldots, n\}$ the mapping $L^{k}: H^{n-k}(X) \rightarrow H^{n+k}(X)$ is surjective.

This result may be obtained in a more direct way by finding a correspondence of the type considered in Proposition 9. For a fixed orbifold structure $\left\{\left(V_{i}, \mathcal{G}_{i}, \pi_{i}\right)\right\}$ on $X$ that satisfies the condition that the associated covering $\mathcal{U}$ is closed under intersections we consider the manifold $N=\bigsqcup_{i} V_{i}$. Let $\tilde{s}$ be a lift of $s: X \rightarrow \bigwedge^{k} T^{*} X$, that is a $k$-form. Then $\tilde{s}$ corresponds to the form

$$
\left.\sum_{i} \tilde{s}\right|_{V_{i}} \in \bigoplus_{i} \Omega^{k}\left(V_{i}\right)=\Omega^{k}(N) .
$$

Proposition 17. The mapping

$$
\left.\Omega^{*}(X) \ni s \rightarrow \sum_{i} \tilde{s}\right|_{V_{i}} \in \Omega^{k}(N)
$$

is a chain isomorphism onto a subcomplex $\Omega_{\Gamma}^{k}(N)$ consisting of forms invariant under the pseudogroup $\Gamma$ generated by the injections

$$
\left(V_{i}, \mathcal{G}_{i}, \pi_{i}\right) \rightarrow\left(V_{j}, \mathcal{G}_{j}, \pi_{j}\right)
$$

and the action of each $\mathcal{G}_{i}$ on $V_{i}$.

Proposition 17 together with Theorem 3 now imply Theorem 16.

REMARK 18. For compact Kähler orbifolds the hard Lefschetz theorem, which is condition (2) of Theorem 16, was demonstrated in [21]. Hence Theorem 1 follows.

\section{References}

[1] D. Alekseevsky, A. Kriegl, M. Losik and P. Michor, 'The Riemannian geometry of orbit spacesthe metric, geodesics, and integrable systems', Publ. Math. Debrecen 62 (2003), 247-276.

[2] J.-L. Brylinski, 'A differential complex for Poisson manifolds', J. Differential Geom. 28 (1988), 93-114.

[3] W. Chen and Y. Ruan, 'Orbifold Gromov-Witten theory', in: Orbifolds in Mathematics and Physics (American Mathematical Society, Providence, RI, 2002), pp. 25-85.

[4] D. Domínguez, 'Finiteness and tenseness theorems for Riemannian foliations', Amer. J. Math. 120 (1998), 1237-1276.

[5] A. El Kacimi-Alaoui and G. Hector, 'Décomposition de Hodge basique pour un feuilletage Riemannien', Ann. Inst. Fourier (Grenoble) 36 (1986), 207-227.

[6] M. Fernández, R. Ibáñez and M. de León, 'On a Brylinski conjecture for compact symplectic manifolds', Quaternionic Structures in Mathematics and Physics, Trieste, 1994 (SISSA, Trieste, 1998), pp. 119-126.

[7] J. Girbau, A. Haefliger and D. Sundararaman, 'On deformations of transversely holomorphic foliations', J. reine angew. Math. 345 (1983), 122-147.

[8] M. Goresky and R. MacPherson, 'Intersection homology theory', Topology 19 (1980), 135-162.

[9] P. Griffiths and J. Harris, Principles of Algebraic Geometry (Wiley, New York, 1978). 
[10] M. Józefowicz and R. Wolak, 'A few remarks on the geometry of the space of leaf closures of a Riemannian foliation', Geometry and Topology of Manifolds, Banach Center Publications, 76 (Institute of Mathematics, Polish Academy of Sciences, Warszawa, 2007), pp. 395-409.

[11] X. Masa, 'Duality and minimality in Riemannian foliations', Comment. Math. Helv. 67 (1992), $17-27$.

[12] O. Mathieu, 'Harmonic cohomology classes of symplectic manifolds', Comment. Math. Helv. 70 (1995), 1-9.

[13] I. Moerdijk and J. Mrčun, Introduction to Foliations and Lie Groupoids (Cambridge University Press, Cambridge, 2003).

[14] P. Molino, Riemannian Foliations (Birkhäuser, Boston, 1988), translated by G. Cairns.

[15] H. K. Pak, 'Transversal harmonic theory for transversally symplectic flows', J. Aust. Math. Soc. 84 (2008), 233-245.

[16] N. Poncin, F. Radoux and R. Wolak, 'A first approximation for quantization of singular spaces', J. Geom. Phys. 59 (2009), 503-518.

[17] H. Rummler, 'Quelques notions simples en géométrie riemannienne et leurs applications aux feuilletages compacts', Comment. Math. Helv. 54 (1979), 224-239.

[18] I. Satake, 'On a generalization of the notion of manifold', Proc. Natl. Acad. Sci. USA 42 (1956), 359-363.

[19] W. Thurston, The geometry and topology of three-manifolds, available at http://www.msri.org/publications/books/gt3m/ 2002.

[20] P. Tondeur, Geometry of Foliations (Birkhäuser, Boston, 1997).

[21] Z. Z. Wang and D. Zaffran, 'A remark on the Hard Lefschetz theorem for Kähler orbifolds', Proc. Amer. Math. Soc. 137 (2009), 2497-2501.

[22] R. Wolak, 'Foliated and associated geometric structures on foliated manifold', Ann. Fac. Sci. Toulouse Math. (5) 10 (1989), 337-360.

[23] D. Yan, 'Hodge structure on symplectic manifold', Adv. Math. 120 (1996), 143-154.

\section{BAK, Institute of Mathematics, Jagiellonian University, Krakow, Poland e-mail: lukasz.bak@im.uj.edu.pl}

A. CZARNECKI, Institute of Mathematics, Jagiellonian University, Krakow, Poland e-mail: andrzej.czarnecki@im.uj.edu.pl 